\title{
Incidental Lymphoplasmacytic Lymphoma Diagnosed Following Robotic-Assisted Laparoscopic Prostatectomy for Prostate Cancer
}

\author{
Omar El-Tajia Altan Omer $^{\mathrm{a}} \quad$ Abdullah Al-Mitwalli A $^{\mathrm{b}} \quad$ Samita Agarwalc \\ Anand Sharma $^{d} \quad$ Nikhil Vasdeva $^{\text {a }}$ \\ aDepartment of Urology, Lister Hospital, Stevenage; bepartment of Urology, St James University Hospital, Leeds; \\ 'Department of Histopathology, Lister Hospital, Stevenage; ${ }^{\mathrm{d} D e p a r t m e n t}$ of Oncology, Mount Vernon Cancer Centre, Middlesex, UK
}

\section{Key Words}

Prostate Cancer • Robotic assisted laparoscopic prostatectomy $\cdot$ Lymphoma

\begin{abstract}
We report a case of prostatic lymphoma of the Waldenström's macroglobulinemia subtype in a 64-year-old gentleman who underwent a robotic-assisted laparoscopic prostatectomy following lower urinary tract symptoms and high grade adenocarcinoma on transperineal prostate biopsy's. Histopathological and immunohistochemistry analysis at the time of surgery was consistent with a CD5-negative small B-cell lymphoma. To our knowledge this is the first reported prostatic lymphoma identified following robotic-assisted laparoscopic prostatectomy and the first documented case of lymphoplasmacytic lymphoma involving prostate. Lymphoma of the prostate is an uncommon entity in surgical practice and their diagnosis often poses considerable difficulty as they often mimic carcinoma. We discuss this rare diagnosis and review the literature for current considerations and prognosis.

(c) 2019 The Author(s)

Published by S. Karger AG, Basel
\end{abstract}

\section{Introduction}

Adenocarcinoma of the prostate is still the most common histological subtype accounting for $>90 \%$ of cases, with lymphoma of the prostate accounting for $<1 \%$ [1].

\section{KARGER}

Fax +4161306 1234

E-Mail karger@karger.com

www.karger.com
(C) 2019 The Author(s)

Published by S. Karger AG, Basel Upen access

This article is licensed under the Creative Commons AttributionNonCommercial-NoDerivatives 4.0 International License (CC BY NC-ND) (http://www.karger.com/Services/OpenAccessLicense) Usage and distribution for commercial purposes as well as any distribution of modified material requires written permission.
The most common subtype of lymphoma appears to be diffuse large B-cell non-Hodgkin's lymphoma (NHL). Currently, radical prostatectomy is considered the gold standard for local treatment of organ-confined prostate cancer. Nodal involvement can be challenging to detect prior to lymph node dissection as it is frequently microscopic and therefore undetectable using existing imaging modalities. Consequently lymph node involvement is often neglected only to be discovered post-operatively by histopathological examination of the resected specimen.

Lymphoplasmacytic lymphoma (Waldenström macroglobulinemia) is an exceptionally rare B-cell disorder secondary to the accumulation of clonally related lymphoplasmacytic cells in the bone marrow, with an incidence of 3 cases per million yearly.

We report a 64-year-old gentleman who presented with lower urinary tract symptoms and to our knowledge is the first documented diagnosis of prostatic lymphoma following robotic-assisted laparoscopic prostatectomy.

\section{Case Presentation}

A 64-year-old gentleman was referred to the urology department with obstructive lower urinary tract symptoms namely nocturia, increased daytime frequency and urgency with a PSA of $11.06 \mathrm{ng} / \mathrm{ml}$ (normal 0-4 ng/ml). Review of his systems was negative and there was no family history of prostate cancer. On examination he had a soft abdomen and digital rectal exam demonstrated a $40 \mathrm{ml}$ benign feeling prostate. An initial laboratory test revealed a hemoglobin of $102 \mathrm{~g} / \mathrm{dl}$ (normal 13.5-17.5 g/dl), WBC of 7.0 $10^{9} / 1$ (normal $\left.3.5-10.5 \times 10^{9} / 1\right)$, platelet count of $253 \times 10^{9} / 1$ (nor- 


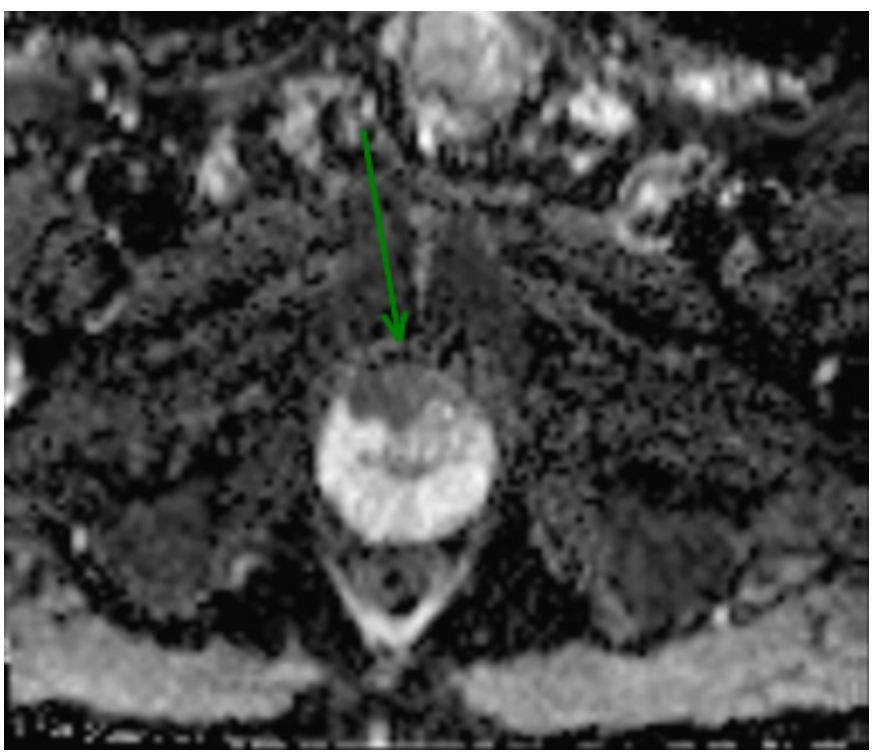

Fig. 1. MRI prostate demonstrating a $25 \times 13 \mathrm{~mm}$ area of ill-defined T2 hypointense change in the anterior gland apex with a PI-RADS score 5/5.

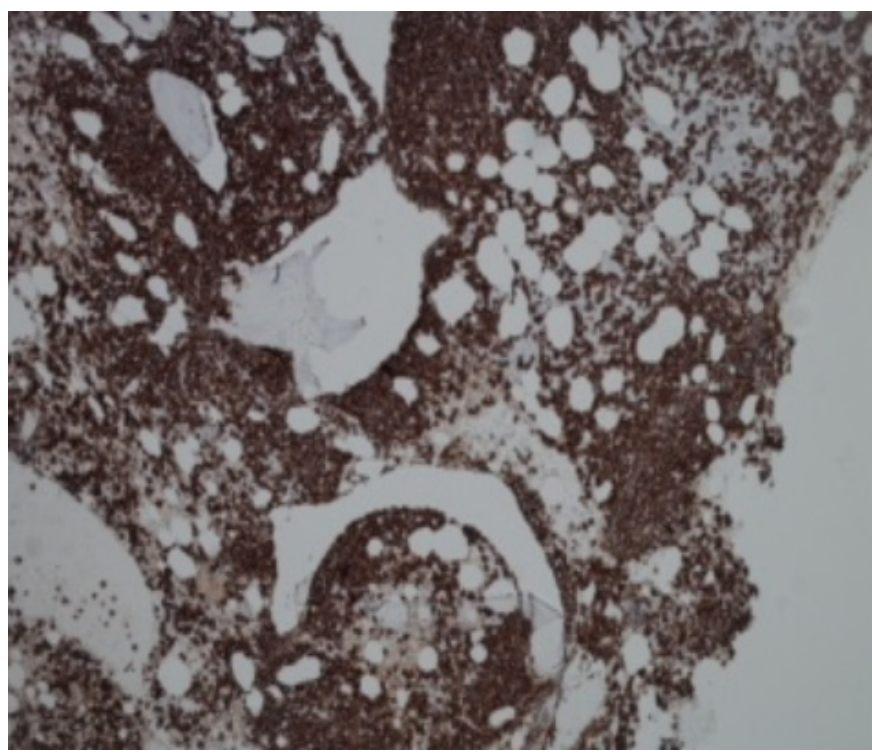

Fig. 2. CD 20 positive lymphoid cells.

\section{Bone Marrow Trephine Examination}

A good core of hypercellular bone marrow (90\% cellularity) showed a predominantly paratrabecular infiltrate of small lymphocytes together with plasmacytoid and plasma cells. The lymphocytes were CD79+, CD20+, Bcl2+, but negative for CD5, CD23 and CD10. The admixed plasma cells were CD138+ with kappa light chain restriction but negative for CD56. There was little trilineage hemopoietic reserve. The features were those of lymphoplasmacytic lymphoma extensively involving the bone marrow.

\section{Molecular Studies}

Molecular studies confirmed the presence of MYD88 muta-

A bilateral, multifocal microacinar type of prostatic adenocarcinoma was identified with a dominant tumor nodule of $33 \mathrm{~mm}$ at the anterior gland. The distal apical margin was negative for tumor involvement and no extra prostatic disease was identified. The lymph nodes showed effacement of the normal architecture by a diffuse infiltrate of small lymphoid cells. Seminal vesicles of the prostate gland demonstrated a patchy infiltrate by a monotonous population of small B-cells showing similar morphological features to those noted in the lymph node. A final Gleason score of $3+4=7$ and stage of pT2c N0 MX is found. It was also necessary for further evaluation by immunohistochemistry.

\section{Immunohistochemistry}

Immunohistochemical staining showed the lymphoid infiltrate was positive for CD20 (fig. 2), CD79a and Bcl2. Staining for light chains showed kappa restricted expression. Negative staining for CD3, CD5, CD23, cyclin D1, CD10, Bcl6, CD21, TdT and CD138 is found. Staining for MIB-1 demonstrated a very low proliferation fraction $(<5 \%)$. Overall the features were those of a CD5-negative small B-cell lymphoma.

Lymphoblastic Lymphoma Following

Robotic Assisted Laparoscopic

Prostatectomy tion.

\section{Further Management}

Final diagnosis was Gleason 7 adenocarcinoma of the prostate with an incidental additional diagnosis of CD5 negative B-cell lymphoplasmacytic lymphoma (Waldenström's macroglobulinemia) identified in the prostate. This gentleman was subsequently commenced on rituximab and bendamustine.

\section{Discussion}

Lymphoplasmacytic lymphoma (Waldenström macroglobulinemia) is a lymphoproliferative disorder belonging to the NHL category characterized by proliferating lymphoplasmacytic components of bone marrow and the presence of monoclonal immunoglobulins (IgM). It is a 
rare disease accounting for $<2 \%$ of NHL [2]. Clinical presentation is frequently heterogeneous while signs and symptoms of organ infiltration can be demonstrated. Despite its indolent course, prompt treatment is necessary to avoid organ impairment.

Its discreet pathology means that incidental findings of NHL are not often recognized at the time of radical prostatectomy for prostate cancer. Lymphoma of the prostate is an uncommon entity in surgical practice. They have been demonstrated to account for $<1 \%$ of prostatic disease. Early studies by Terris et al. [1] reported 1,092 prostatectomies of which only $0.8 \%$ of cases demonstrated a lymphoma. They concluded that the management of the majority of incidental hematological malignancies can be expectant with only aggressive lymphoma subtypes needing radical therapy.

Larger studies by Weir et al. [3] which looked at 6,143 patients revealed lymphocytic lymphoma in $0.3 \%$ of specimens. One patient presented with submandibular swelling and elevated WBC with the other patients all presenting with no overt features of lymphoma. Moreover similar to in our case, imagining studies failed to demonstrate any disease in the pelvis and the characteristical features which are often typical of a lymphoma were not seen in the majority of cases. The important point to elaborate on here is that lymphoma can be easily missed if careful attention is not given.

Numerous case reports and series have reported good prognosis following the incidental diagnosis of hematological malignancies following radical prostatectomy. Di
Meglio et al. [4] reported after 30 months there was no evidence of either lymphoma recurrence or of prostate cancer progression (undetectable serum PSA) in a patient diagnosed with stage IV lymphoma after chemotherapy treatment (EBVD regimen). Although uncommon, early detection of lymphoma after radical prostatectomy will allow optimal management.

In a more recent case report by Drinis et al. [5], they describe an incidental NHL diagnosed after radical prostatectomy for a prostate adenocarcinoma. Lymphadenectomy at the time revealed a low grade lymphoma. Five year follow-up demonstrated no disease progression for both his lymphoma and prostate adenocarcinoma.

\section{Conclusion}

Although rare, prostatic lymphoma can present much like a typical adenocarcinoma of the prostate. The literature suggests that lymphoma involving the prostate generally has a good prognosis yet management following radical prostatectomy varies with each subtype. This is certainly the first case of lymphoma involving the prostate both at our hospital in over 1,150 robotic prostatectomies now performed and in the literature. Moreover, to our knowledge this is the first documented case of lymphoplasmacytic lymphoma involving prostate.

\section{References}

1 Terris MK, Hausdorff J, Freiha FS: Hematolymphoid malignancies diagnosed at the time of radical prostatectomy. J Urol 1997:158:1457-1459.

2 Swerdlow SH, Campo E, Pileri SA, Harris NL, Stein H, Siebert R, Advani R, Ghielmini M, Salles GA, Zelenetz AD, Jaffe ES: The 2016 revision of the World Health Organization classification of lymphoid neoplasms. Blood 2016;127:2375-2390.
3 Weir EG, Epstein JI: Incidental small lymphocytic lymphoma/chronic lymphocytic leukemia in pelvic lymph nodes excised at radical prostatectomy. Arch Pathol Lab Med 2003; 127:567-572.

4 Di Meglio A, Nuzzo PV, Ricci F, Spina B, Boccardo F: Incidental advanced-stage Hodgkin lymphoma diagnosed at the time of radical prostatectomy for prostatic cancer: a case report and review of literature. BMC Cancer 2014;14:613.
5 Drinis S, Finkelstein MP, Tortorelis DG, Konno S, Choudhury MS: Five-year prognosis after radical prostatectomy in a patient with localized prostate cancer and incidental non-Hodgkin's lymphoma. Urol Int 2001;66:105-107. 